\title{
Clinical presentation of infants hospitalised with pertussis
}

\author{
G Kahl, ${ }^{1}$ MB BCh, DCH, MMed (Paed), FC Paed (SA); \\ U M Hallbauer, ${ }^{1}$ MB BCh, MPraxMed, DCH, MMed (Paed), FC Paed (SA); G Joubert, ${ }^{2}$ BA, MSc \\ ${ }^{1}$ Department of Paediatrics and Child Health, Faculty of Health Sciences, University of the Free State, Bloemfontein, South Africa \\ ${ }^{2}$ Department of Biostatistics, Faculty of Health Sciences, University of the Free State, Bloemfontein, South Africa
}

Correspondingauthor: G Kahl (gkahl11383@yahoo.com)

\begin{abstract}
Background. Despite the widespread use of pertussis vaccine, there has been a resurgence of pertussis cases in developed and developing countries. South Africa lacks data regarding clinical presentation and healthcare impact of pertussis.

Objectives. To describe the clinical presentation and healthcare impact in hospitalised infants with confirmed pertussis.

Methods. This was a retrospective cohort study, conducted in Bloemfontein between April 2008 and September 2012. Infants with laboratory-confirmed pertussis (group $1 ; N=102$ ), were compared with infants with a negative pertussis result (group 2; $N=104$ ) and infants with a lower respiratory tract infection of unspecified aetiology (group 3; $N=104$ ). The following data were extracted from the clinical records: demographics, presenting symptoms, paediatric intensive care unit (PICU) admission, length of stay in the general ward and PICU, overall hospital stay and outcome.

Results. There were no significant demographic differences between the groups. A larger percentage of infants in group 1 ( $n=41,40 \%)$ required PICU admission compared with group $2(n=37,36 \%)$ and group $3(n=20,19 \%)$. The median PICU stay of group 1 was longer (11 days) compared with group 2 (6 days) and group 3 ( 5 days). The presence of cough and post-tussive vomiting was significantly higher in group 1 than groups 2 and 3 . There was no significant difference in mortality between the groups.

Conclusion. Pertussis results in significant morbidity in infants. Measures to identify and manage this vaccine-preventable disease should be considered at a national level.
\end{abstract}

S Afr J Child Health 2016;10(3):176-180. DOI:10.7196/SAJCH.2016.v10i3.1115

Pertussis, commonly known as whooping cough, is an acute respiratory illness caused by Bordetella pertussis, a Gram-negative coccobacillus restricted to humans. Although the disease affects all age groups, the disease is most serious in infants ( $<6$ months of age) and in incompletely immunised children. ${ }^{[1]}$ There are $\sim 50$ million cases of pertussis and 300000 pertussis-related deaths annually worldwide according to the World Health Organization (WHO) ${ }^{[2]}$ Data from South Africa (SA) are incomplete. Only patients ill enough to present to hospital are investigated, so available reports are an underestimate of the true number of pertussis cases in SA. ${ }^{[3]}$ Vaccinations have had a major impact on the number of pertussis cases worldwide. ${ }^{[4]}$ The WHO estimates that 687000 pertussis deaths are prevented annually by global vaccination. ${ }^{[5]}$ Vaccination reduces the severity of clinical disease as well as the duration of coughing, transmission and rate of hospitalisation. ${ }^{[6]}$ Children aged 6 - 24 months who have not received any dose of pertussis vaccine are at a ten-fold risk of hospitalisation compared with those who have been partially or fully immunised. ${ }^{[7]}$ In SA, whole-cell pertussis vaccine (administered as a trivalent vaccine to include tetanus and diphtheria) was replaced by acellular pertussis vaccine in 2009. The pentavalent vaccine (diphtheria, tetanus, acellular pertussis, inactivated polio vaccine and haemophilus influenza type b (DTaPIPV/Hib) was also introduced as part of the Expanded Programme of Immunisation (EPI). ${ }^{[8]}$ This vaccine is administered at the ages of 6,10 and 14 weeks, and a booster dose is given at 18 months. There is no booster for pertussis after 18 months. Infants $<18$ weeks are still considered to be incompletely vaccinated as they have not yet developed full immunity in response to the pertussis vaccine. ${ }^{[9]}$ According to the EPI, no booster dose is given at school-going age or adolescence. Adolescents and adults play an important role in spreading pertussis to infants. ${ }^{[10]}$ The immunisation of adolescents and adults, especially pregnant women, not only protects them from disease but potentially diminishes its spread to young, vulnerable infants. Some developed countries have introduced preschool and adolescent boosters into their vaccination programmes, as well as maternal third-trimester immunisations. ${ }^{[11]}$

Currently, pertussis is the only vaccine-preventable disease with an increasing incidence globally. ${ }^{[12]}$ Data on the burden of pertussis in developing countries are scarce, particularly in Africa. The morbidity and mortality of pertussis in SA, and the impact that it has on the healthcare system, are unknown. The objective of this study was to describe the clinical presentation of and healthcare impact in infants with pertussis in the public health sector in Bloemfontein, SA. The demographic data, presenting symptoms, HIV and nutritional status, prematurity at birth, length of stay in the general ward and paediatric intensive care unit (PICU) and mortality rate were compared between three groups of patients - those with confirmed pertussis, those tested for pertussis but with a negative result, and those with a lower respiratory tract infection (LRTI) of unspecified aetiology.

\section{Methods}

This was a retrospective cohort study. Hospitalised infants $<12$ months who were admitted with an LRTI between April 2008 and September 2012 (54 months) were considered for the study. The public sector hospitals were Universitas Hospital (tertiary hospital), Pelonomi Hospital (regional hospital) and National District Hospital (district hospital), which form the academic complex in Bloemfontein. The data were collected according to three groups: proven pertussis disease, proven negative for pertussis and LRTI of unspecified aetiology. Each study group included patients from Pelonomi Hospital and National District Hospital, where they were initially admitted. If required, intensive care was provided at Pelonomi Hospital and/or 
Universitas Hospital. The period for inclusion of cases was started when the first case had been identified in Bloemfontein (April 2008) until start of the study (September 2012).

\section{Group 1: proven positive for pertussis}

In these patients, a nasopharyngeal swab was taken and tested for pertussis using a polymerase chain reaction (PCR) test, specifically targeting the IS481 sequence. Attending doctors requested a pertussis swab at their clinical discretion, but they were also made aware of the suggestive features of pertussis: post-tussive vomiting, apnoea, coughing for more than 2 weeks and a white cell count of more than $20 \times 10^{9} / \mathrm{L}$. The swab was taken either on admission or during the hospital stay. The National Health Laboratory Services (NHLS) databank was used to identify all PCR pertussis swabs taken during the specified period in infants $<12$ months. Only laboratoryconfirmed pertussis-positive cases were included in this group. Of the 117 patients identified by the NHLS with a pertussis PCR-positive result, the medical records of 13 cases could not be traced, and 2 cases were excluded as they did not meet the study criteria (one patient was not admitted, and the other had a pertussis PCR swab done after discharge). A total of 102 patients with proven pertussis disease were thus included in the group (Fig. 1).

\section{Group 2: proven negative for pertussis}

This group included infants who had been tested for pertussis but were pertussis PCR negative. The NHLS indicated that 405 cases had a negative PCR pertussis swab result. From this group, 104 cases were randomly selected to equal the number of patients in group 1 (Fig. 1). This was done prior to the exclusion of two patients who did not meet study criteria in group 1 . The random.org online software program (Ireland) was used for this purpose. ${ }^{[13]}$

\section{Group 3: LRTI of unspecified aetiology}

Infants with an LRTI were identified retrospectively using statistics that are kept of all the paediatric admissions to Pelonomi Hospital

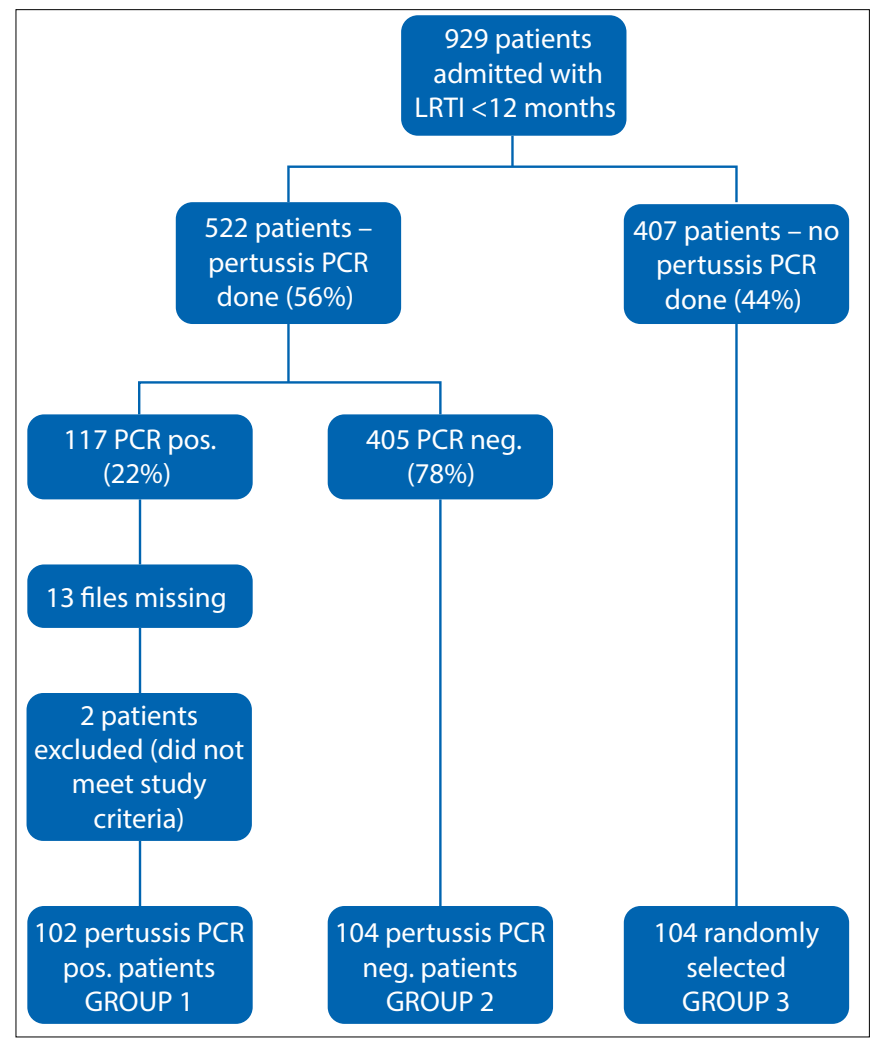

Fig. 1. Selection of cases for inclusion in the study. and National District Hospital. A total of 929 patients were identified, and 104 were randomly selected to equal the number of patients in groups 1 and 2, using the same random selector as for group 2 (Fig. 1). Group 3 was seen as a control group for groups 1 and 2, and represented infants from the general population with an LRTI with the same background risk of pertussis.

The following demographic and clinical data were obtained from the patients' hospital records: date of birth (age), gender and whether birth was premature (gestational age $<37$ weeks), and details of presenting symptoms (presence of cough and duration thereof, post-tussive vomiting, whoop, fever or apnoea). The patients' HIV and nutritional status was documented. Undernutrition was defined using the WHO charts (2009) and included infants with a weight for length between -2 and $-3 z$-score or $<-3 z$-score. The dates of hospital admission and discharge or death were recorded, and if applicable, date of PICU admission and discharge or death. The outcome survival or death - was also recorded. The data were entered into a study database by the Department of Biostatistics. Results were summarised by frequencies, percentages, means, standard deviations (SDs) and percentiles. The tests used to determine the statistical significance were the Kruskall-Wallis and Mann-Whitney tests for numerical data, and $\chi^{2}$ or Fisher's exact test for categorical data.

\section{Research ethics committee approval}

The study was approved by the Ethics Committee of the Faculty of Health Sciences of the University of the Free State (ECUFS no.: 216/2012). Written permission to conduct the study was obtained from the Head of Clinical Services of each hospital.

\section{Results}

There were no significant differences between the groups with regard to gender, age and gestational age at birth (Table 1). The presence of cough and post-tussive vomiting was significantly higher in the pertussis-positive group compared with the pertussis-negative group and the LRTI group. The median duration of cough was 3 days for all groups (range 1 - 28 days). The presence of apnoea was significantly higher in the pertussis-positive group compared with both the pertussis-negative and LRTI groups. The presence of fever was comparable between all the groups. Only one infant with pertussis had whoop as a presenting symptom (Table 1). A greater proportion of patients in the pertussis-positive group compared with either the pertussis-negative or LRTI group were HIV-positive ( $n=25$ (28.7\%); $n=20$ (20.0\%); $n=12$ (12.1\%), respectively). Among the HIV-positive infants (including undernourished, well-nourished and infants born prematurely), the length of stay in the ward, PICU and overall hospital stay were not significantly different between the three groups (Table 2). There was a higher rate of undernutrition in the pertussis-positive group compared with the pertussis-negative or the LRTI groups $(n=47$ (49.0\%); $n=34$ (33.7\%); $n=32$ (31.7\%)). This group included HIV-positive and HIV-negative infants. Among the undernourished infants, the length of stay in the ward, PICU and overall hospital stay were not significantly different between the pertussis-positive, pertussis-negative and LRTI groups (Table 2). There was no difference in rates of prematurity between the three groups $(n=28$ (29.2\%); $n=29$ (29.3\%); $n=29$ (29.0\%)). The median hospital stay among children born prematurely was longest among the pertussis-positive group and shortest for those with an LRTI of unspecified aetiology (Table 2). Among HIV-negative and wellnourished infants, the length of stay in the ward and PICU was not significantly different between the three groups (Table 3 ). The hospital stay between the pertussis-positive and pertussis-negative groups was not significantly different (median 8 days v. 7 days, respectively; $p=0.19$ ). However, there was a significant difference in hospital stay between the three groups in total, namely between the pertussis-positive (median 8 days) and the LRTI group (median 
Table 1. Baseline data of the pertussis-positive, pertussis-negative and LRTI groups regarding demographic profile and presenting symptoms ${ }^{\star}$

\begin{tabular}{|c|c|c|c|c|c|c|c|}
\hline & $\begin{array}{l}\text { Pertussis-positive } \\
\text { group (group 1) } \\
(N=102)\end{array}$ & $\begin{array}{l}\text { Pertussis-negative } \\
\text { group (group 2) } \\
(N=104)\end{array}$ & $\begin{array}{l}\text { LRTI group } \\
\text { (group 3) } \\
(N=104)\end{array}$ & $\begin{array}{l}\text { Group } 1 \text { v. } 2 \\
p \text {-value }\end{array}$ & $\begin{array}{l}\text { Group } 2 v . \\
3 p \text {-value }\end{array}$ & $\begin{array}{l}\text { Group } 1 \mathrm{v} \text {. } \\
3 p \text {-value* }\end{array}$ & $\begin{array}{l}\text { Overall } \\
p \text {-value }\end{array}$ \\
\hline \multicolumn{8}{|l|}{ Characteristics } \\
\hline Age (weeks), median (IQR) & $10.0(6-23)$ & $13.5(6-24)$ & $12.0(7-26)$ & - & - & - & 0.27 \\
\hline Gender, $n(\%)$ & & & & & & & 0.07 \\
\hline Male & $44(43.1)$ & $50(48.1)$ & $61(58.7)$ & - & - & - & \\
\hline Female & $58(56.9)$ & $54(51.9)$ & $43(41.4)$ & - & - & - & \\
\hline \multicolumn{8}{|l|}{ Presence of symptoms, $n(\%)$} \\
\hline Cough & $94(95.9)$ & $86(82.7)$ & $89(85.6)$ & $<0.01$ & 0.57 & 0.01 & - \\
\hline Post-tussive vomiting & $40(42.1)$ & $16(17.2)$ & $13(12.9)$ & $<0.01$ & 0.40 & $<0.01$ & $<0.01$ \\
\hline Whoop & $1(1.0)$ & $0(0)$ & $1(1.0)$ & - & - & - & 0.77 \\
\hline Fever & $42(43.3)$ & $43(41.4)$ & $48(46.2)$ & - & - & - & 0.78 \\
\hline Apnoea & $19(19.0)$ & $13(12.5)$ & $7(6.7)$ & 0.20 & 0.16 & 0.01 & 0.03 \\
\hline
\end{tabular}

Table 2. Comparison of median length of stay in the ward, PICU and overall hospital stay between the pertussis-positive, pertussisnegative and LRTI groups in HIV-positive and undernourished infants and those with a history of prematurity

\begin{tabular}{|c|c|c|c|c|}
\hline & $\begin{array}{l}\text { Pertussis-positive } \\
\text { group (group 1) }\end{array}$ & $\begin{array}{l}\text { Pertussis-negative } \\
\text { group (group 2) }\end{array}$ & $\begin{array}{l}\text { LRTI group } \\
\text { (group 3) }\end{array}$ & $\begin{array}{l}\text { Overall } \\
p \text {-value }\end{array}$ \\
\hline HIV-positive infants $(N=87), n(\%)^{*}$ & $25 / 87(28.7)$ & $20 / 100(20.0)$ & $12 / 99(12.1)$ & 0.02 \\
\hline Duration of ward stay (days), median (IQR) & $\begin{array}{l}(n=25) \\
9(6-15)\end{array}$ & $\begin{array}{l}(n=20) \\
12(9-24)\end{array}$ & $\begin{array}{l}(n=12) \\
11(7.5-22.5)\end{array}$ & 0.31 \\
\hline Duration of PICU stay (days), median (IQR) & $\begin{array}{l}(n=12) \\
11.5(7-16)\end{array}$ & $\begin{array}{l}(n=8) \\
5.5(3.5-12)\end{array}$ & $\begin{array}{l}(n=3) \\
9(3-33)\end{array}$ & 0.34 \\
\hline $\begin{array}{l}\text { Duration of hospital stay (ward and PICU) (days), } \\
\text { median (IQR) }\end{array}$ & $\begin{array}{l}(n=25) \\
14(9-22)\end{array}$ & $\begin{array}{l}(n=20) \\
14(11.5-28.5)\end{array}$ & $\begin{array}{l}(n=12) \\
11(7.5-24.5)\end{array}$ & 0.61 \\
\hline Undernourished infants, $n(\%)^{*}$ & $47 / 96(49.0)$ & $34 / 101(33.7)$ & $32 / 101(31.7)$ & 0.02 \\
\hline Duration of ward stay (days), median (IQR) & $\begin{array}{l}(n=47) \\
9(7-16)\end{array}$ & $\begin{array}{l}(n=34) \\
8.5(6-21)\end{array}$ & $\begin{array}{l}(n=32) \\
7(5-13.5)\end{array}$ & 0.26 \\
\hline Duration of PICU stay (days), median (IQR) & $\begin{array}{l}(n=19) \\
7(5-13)\end{array}$ & $\begin{array}{l}(n=15) \\
7(4-12)\end{array}$ & $\begin{array}{l}(n=11) \\
6(4-10)\end{array}$ & 0.81 \\
\hline $\begin{array}{l}\text { Duration of hospital stay (ward and PICU) (days), } \\
\text { median (IQR) }\end{array}$ & $\begin{array}{l}(n=47) \\
12(8-21)\end{array}$ & $\begin{array}{l}(n=34) \\
12(7-26)\end{array}$ & $\begin{array}{l}(n=32) \\
8.5(7-14.5)\end{array}$ & 0.13 \\
\hline Infants with history of prematurity, $n(\%)^{*}$ & $28 / 96(29.2)$ & $29 / 99(29.3)$ & $29 / 100(29.0)$ & 1.00 \\
\hline Duration of ward stay (days), median (IQR) & $\begin{array}{l}(n=28) \\
8(4-10.5)\end{array}$ & $\begin{array}{l}(n=29) \\
8(6-16)\end{array}$ & $\begin{array}{l}(n=29) \\
6(4-8)\end{array}$ & 0.10 \\
\hline Duration of PICU stay (days), median (IQR) & $\begin{array}{l}(n=14) \\
9(5-11)\end{array}$ & $\begin{array}{l}(n=15) \\
8(6-13)\end{array}$ & $\begin{array}{l}(n=8) \\
4.5(3-9)\end{array}$ & 0.17 \\
\hline $\begin{array}{l}\text { Duration of hospital stay (ward and PICU) (days), } \\
\text { median (IQR) }\end{array}$ & $\begin{array}{l}(n=28) \\
10.5(8-15.5)\end{array}$ & $\begin{array}{l}(n=29) \\
13(8-21)\end{array}$ & $\begin{array}{l}(n=29) \\
7(4-9)\end{array}$ & $<0.01$ \\
\hline
\end{tabular}

6 days; $p<0.01$ ), and between the pertussis-negative (median 7 days) and LRTI group (median 6 days; $p=0.01$ ). Forty-one patients $(40 \%)$ with confirmed pertussis were admitted to PICU compared with $37(36 \%)$ patients in the pertussis-negative group $(p=0.49)$ and 20 (19\%) patients in the LRTI group $(p<0.01 ; p=0.01$ between pertussisnegative and LRTI groups). Median PICU stay was significantly longer in the confirmed pertussis group compared with both the pertussisnegative group $(p=0.02)$ and the LRTI group $(p=0.01)$. The length of general ward stay was longer in both the confirmed pertussis and pertussis-negative groups compared with the LRTI group. Overall median hospital (PICU plus general ward) stay was significantly longer in the pertussis-positive group compared with both the pertussis-negative $(p=0.05)$ and LRTI groups $(p<0.01)$ (Table 4$)$. There was no statistically significant difference in mortality between the three groups. In both the confirmed pertussis group and the LRTI group, seven patients died (6.8\% and $6.7 \%$, respectively), and three 
patients died in the pertussis-negative group (2.9\%). In the confirmed pertussis group, six of the seven patients $(85.7 \%)$ who died were $<6$ months of age, and five of the seven $(71.4 \%)$ were $<3$ months old. More patients were diagnosed with pertussis in the months between January and April of each year. There was also a marked rise in incidence of pertussis in the year 2011 (Fig. 2).

\section{Discussion}

Pertussis is associated with considerable morbidity and some mortality, and the recent resurgence of pertussis in developed and developing countries has necessitated the need to evaluate its impact in SA. Scant data are available from SA regarding the morbidity and healthcare burden of pertussis. The data from this study show that $40 \%$ of infants with proven pertussis required PICU admission, compared with only $19 \%$ of infants with an LRTI of unspecified origin, and $36 \%$ of infants who tested negative for pertussis. The death rate was not significantly different between the three groups but was generally very high. The high rates of PICU admission and mortality rates may be a reflection that two of the hospitals (Pelonomi and Universitas) are secondary- or tertiarylevel hospitals that include infants who were too ill to be managed at peripheral hospitals. The length of stay in PICU for confirmed pertussis patients was also far greater than for the pertussis-negative and LRTI groups. General ward stay and overall hospital stay were also significantly prolonged in the group with confirmed pertussis. However, general ward stay and overall hospital stay were also longer in the pertussis-negative group compared with the LRTI group. Perhaps some infants in the pertussis-negative group were tested for pertussis because they had already been admitted in hospital for a long time and were not responding to treatment, and other diagnoses were being explored. In addition, group 2 may have included false-negative cases. A greater proportion of infants with proven pertussis were immune compromised (HIV-positive or undernourished) than infants of either the pertussis-negative or the LRTI of unknown aetiology groups. This may indicate a greater

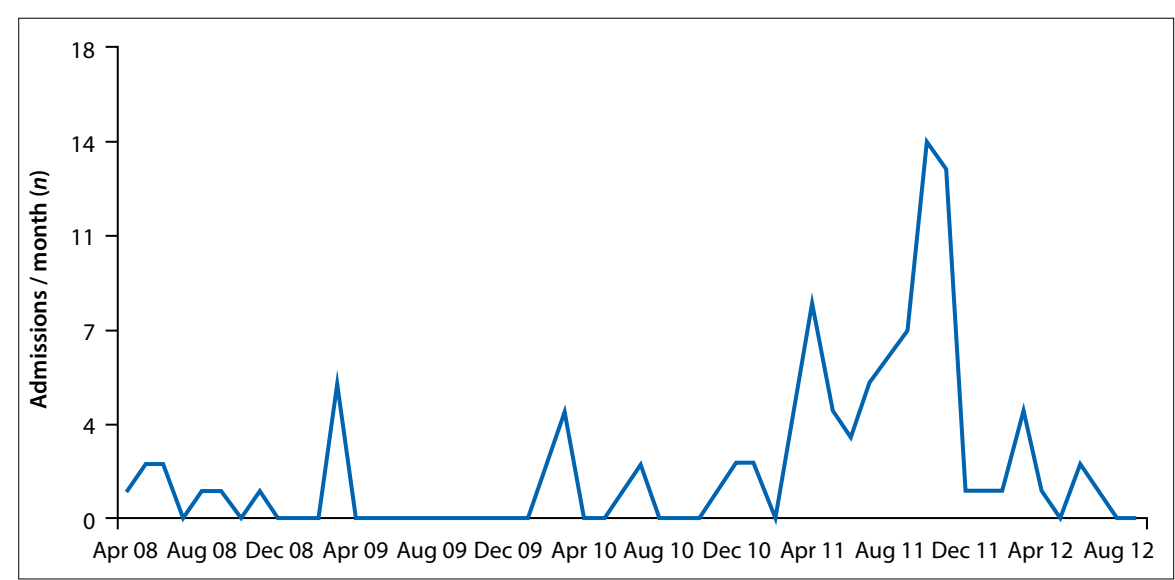

Fig. 2. Pertussis cases in infants per month between April 2008 and September 2012.

Table 3. Comparison of median length of stay in the ward, PICU and overall hospital stay between the pertussis-positive, pertussisnegative and LRTI groups in HIV-negative and well-nourished infants

\begin{tabular}{|c|c|c|c|c|}
\hline & $\begin{array}{l}\text { Pertussis-positive } \\
\text { group (group 1) }\end{array}$ & $\begin{array}{l}\text { Pertussis-negative } \\
\text { group (group 2) }\end{array}$ & $\begin{array}{l}\text { LRTI group } \\
\text { (group 3) }\end{array}$ & $\begin{array}{l}\text { Overall } \\
p \text {-value }\end{array}$ \\
\hline HIV-negative and well-nourished infants, $n$ & 35 & 54 & 64 & \\
\hline Duration of ward stay (days), median (IQR) & $\begin{array}{l}(n=35) \\
6(3-10)\end{array}$ & $\begin{array}{l}(n=54) \\
6(4-8)\end{array}$ & $\begin{array}{l}(n=64) \\
5(4-7)\end{array}$ & 0.39 \\
\hline Duration of PICU stay (days), median (IQR) & $\begin{array}{l}(n=12) \\
9(6-11)\end{array}$ & $\begin{array}{l}(n=16) \\
5(3.5-10)\end{array}$ & $\begin{array}{l}(n=5) \\
4(3-8)\end{array}$ & 0.17 \\
\hline Duration of hospital stay (ward and PICU) (days), median (IQR) & $\begin{array}{l}(n=35) \\
8(6-13)\end{array}$ & $\begin{array}{l}(n=54) \\
7(5-10)\end{array}$ & $\begin{array}{l}(n=64) \\
6(4-8)\end{array}$ & $<0.05$ \\
\hline
\end{tabular}

Table 4. Comparison of median length of stay in the ward, PICU and overall hospital admission between the confirmed pertussispositive, pertussis-negative and LRTI (unspecified aetiology) groups

\begin{tabular}{|c|c|c|c|c|c|c|}
\hline & $\begin{array}{l}\text { Pertussis-positive } \\
\text { group (group 1) } \\
(N=102)\end{array}$ & $\begin{array}{l}\text { Pertussis-negative } \\
\text { group (group 2) } \\
(N=104)\end{array}$ & $\begin{array}{l}\text { LRTI group } \\
\text { (unspecified aetiology) } \\
\text { (group 3) }(N=104)\end{array}$ & $\begin{array}{l}p \text {-value } \\
\text { group } 1 \text { v. } 2\end{array}$ & $\begin{array}{l}p \text {-value } \\
\text { group } 2 \text { v. } 3\end{array}$ & $\begin{array}{l}p \text {-value } \\
\text { group } 1 \text { v. } 3\end{array}$ \\
\hline $\begin{array}{l}\text { Duration of ward stay } \\
\text { (days), median (IQR) }\end{array}$ & $\begin{array}{l}(n=102) \\
8(5-13)\end{array}$ & $\begin{array}{l}(n=104) \\
7(5-11)\end{array}$ & $\begin{array}{l}(n=104) \\
6(4-8)\end{array}$ & 0.47 & 0.01 & $<0.01$ \\
\hline $\begin{array}{l}\text { Duration of PICU stay } \\
\text { (days), median (IQR) }\end{array}$ & $\begin{array}{l}(n=41) \\
11(6-14)\end{array}$ & $\begin{array}{l}(n=37) \\
6(4-11)\end{array}$ & $\begin{array}{l}(n=20) \\
5(3.5-9.5)\end{array}$ & 0.02 & 0.61 & 0.01 \\
\hline $\begin{array}{l}\text { Duration of hospital stay } \\
\text { (ward and PICU) (days), } \\
\text { median (IQR) }\end{array}$ & $\begin{array}{l}(n=102) \\
11(7-20)\end{array}$ & $\begin{array}{l}(n=104) \\
8(6-14.5)\end{array}$ & $\begin{array}{l}(n=104) \\
7(4-9)\end{array}$ & 0.05 & $<0.01$ & $<0.01$ \\
\hline
\end{tabular}


the pertussis-negative group compared with the LRTI group. This reflects that infants affected by pertussis without conditions that contribute to immune compromise are more affected than infants with LRTI of unknown aetiology. The pertussis-negative group may include false-negative infants, and in addition, these infants may have been tested for pertussis because they had been admitted to hospital for a long time and were not improving on treatment, and other diagnoses were being considered. Confirmation of pertussis can be difficult in hospitals with minimal access to laboratories, and considering pertussis on clinical grounds is important. In this study, the presence of cough was the most common symptom, followed by post-tussive vomiting and apnoea. Few infants presented with a fever or whoop. The duration of cough in infants with pertussis in this study was very variable, and if only infants with a cough for $>2$ weeks had been tested for pertussis, as the WHO definition required, ${ }^{[14]}$ many pertussis diagnoses may have been missed. It is therefore important to consider pertussis in infants with a short duration of cough. Bearing in mind the severity of pertussis in infants $<18$ weeks who are incompletely immunised, prevention of, rather than treating, the disease should be a priority. In SA, replacing the current tetanus and diphtheria (Td) vaccine scheduled at the age of 6 years with $\mathrm{DTaP}$, as well as immunisation of adolescents and pregnant women, could be a feasible intervention, as is being done in many countries. ${ }^{[10]}$ Early diagnosis and early appropriate treatment with an antibiotic (macrolide) are important, as these reduce the period of communicability of pertussis, and may also reduce the duration and severity of symptoms, ${ }^{[15]}$ and in turn reduce the length of hospital stay. Close contacts at risk of acquiring severe disease should receive a course of a macrolide as well, as secondary disease is estimated to occur in up to $80 \%$ of household contacts. ${ }^{[16]}$ The patients who tested positive in this study, as well as their mothers, were treated for 7 days with a macrolide (erythromycin or clarithromycin).

\section{Study limitations}

There were several limitations to this study. The technique for collection of the PCR pertussis swab in infants suspected of having pertussis was not predefined, and poor collection technique may have resulted in false-negative results. In addition, not all infants who were admitted to one of the hospitals were tested for pertussis, as testing was only done on clinical suspicion and therefore infants with pertussis may have been missed. Another limitation was that the pertussis immunisation details could not be included nor accurately documented in the analysis record, as this was often not available from the parent or caregiver. Vaccination could have been compared among the three groups. The infants tested for pertussis were generally more ill than the infants with an LRTI of unknown aetiology; the pertussis-positive group may therefore represent a sicker group of pertussis cases, and other less severe cases may not have been tested, with the potential for selection bias.

\section{Conclusion}

Pertussis has re-emerged as a serious illness with a significant morbidity and some mortality, even in high-income countries. Not many data about pertussis are available in SA. Health resources are often scarce, and more information needs to be available regarding pertussis in order to target specific prevention strategies and treatment policies. This study has provided an indication of the impact that pertussis has on healthcare, namely prolonged PICU and overall hospital stay. Coughing for $<2$ weeks does not exclude a diagnosis of pertussis. Whoop is rare in infants with pertussis. HIV infection, undernutrition and prematurity at birth do not worsen the clinical presentation of pertussis. Further research into the epidemiology of pertussis in SA is needed in order to inform national control strategies and vaccination policies. This study from one region of SA provides some insight into the healthcare burden of pertussis.

Acknowledgements. Prof. G Hussey reviewed the draft manuscript and provided valuable suggestions.

\section{References}

1. Gordon M, Davies HD, Gold R. Clinical and microbiological features of children presenting with pertussis to a Canadian pediatric hospital during an elevenyear period. Pediatr Infect Dis J 1994;13(7):617-622. DOI:10.1097/00006454199407000-00007

2. World Health Organization. WHO-recommended surveillance standard of pertussis. http:/apps.who.int/immunization-monitoring/en/globalsummary/ diseaseselect.cfm (accessed 12 February 2014).

3. Archer B, Lowman W, Kularatne R, et al. Laboratory-confirmed pertussis in the public health sector, 2008 - 2011. National Health Laboratory Service, National Institute for Communicable Diseases 2011;9(4):81-83.

4. Cherry JD. Epidemic pertussis in 2012 - the resurgence of a vaccine-preventable disease. N Engl J Med 2012;367(9):785-787. DOI:10.1056/NEJMp1209051

5. World Health Organization. Vaccines and diseases. Pertussis. http://www.who. int/immunization/diseases/pertussis/en (accessed 12 February 2014).

6. Baptista PN, Magalhaes V, Rodrigues LC, et al. Pertussis vaccine effectiveness in reducing clinical disease, transmissibility and proportion of cases with a positive culture after household exposure in Brazil. Pediatr Infect Dis J 2006;25(9):844-846. DOI:10.1097/01.inf.0000232642.25495.95

7. Stojanov S, Liese J, Belohradsky BH. Hospitalization and complications in children under 2 years of age with Bordetella pertussis infection. Infection 2000;28(2):106-110. DOI:10.1007/s150100050056

8. Baker L. The face of South Africa's Expanded Programme on Immunisation (EPI) schedule. S Afr Pharm J 2010;77(1):18-21.

9. Greenberg DP, von König CH, Heininger U. Health burden of pertussis in infants and children. Pediatr Infect Dis J 2005;24(5):S39-43. DOI:10.1097/01. inf.0000160911.65632.e1

10. Pan American Health Organization. Control of diphtheria, pertussis, haemophilus influenzae type b, and hepatitis B: Field guide. Washington DC: Pan American Health Organization, World Health Organization, 2005.

11. Ward JI, Cherry JD, Chang SJ, et al. Efficacy of an acellular pertussis vaccine among adolescents and adults. N Engl J Med 2005;353(15):1555-1563. DOI:10.1056/NEJMoa050824

12. Black S. Epidemiology of pertussis. Pediatr Infect Dis J 1997;16(4):85-89. DOI:10.1097/00006454-199704001-00003

13. Mads Haahr, School of Computer Science and Statistics at Trinity College, Dublin. http://www.random.org/sequences (accessed 15 March 2013).

14. World Health Organization. WHO-recommended surveillance standard of pertussis. www.who.int/.../monitoring_surveillance/burden/vpd/surveillance_ type/passive/pertussis standards/en (accessed 11 February 2011).

15. Tiwari T, Murphy TV, Moran J, National Immunization Program CDC. Recommended antimicrobial agents for the treatment and post exposure prophylaxis of pertussis: 2005 CDC Guidelines. MMWR Recomm Rep 2005;54(RR-14):1-16

16. Schellekens J, Wirsing von König C, Gardner P. Pertussis sources of infection and routes of transmission in the vaccination era. Ped Infect Dis J 2005;24(5):S19-S24. DOI:10.1097/01.inf.0000160909.24879.e6. 